\title{
Comparison of Pulmonary Functions at Onset of Ventilatory Insufficiency in Patients With Amyotrophic Lateral Sclerosis, Duchenne Muscular Dystrophy, and Myotonic Muscular Dystrophy
}

\author{
Han Eol Cho, $\mathrm{MD}^{1}$, Jang Woo Lee, $\mathrm{MD}^{1}$, Seong Woong Kang, $\mathrm{MD}$, $\mathrm{PhD}^{1}$, Won $\mathrm{Ah}$ Choi, MD, $\mathrm{PhD}^{1}$, \\ Hyeonjun $\mathrm{Oh}, \mathrm{MD}^{1}$, Kil Chan Lee, $\mathrm{MD}^{2}$
}

\begin{abstract}
${ }^{1}$ Department of Rehabilitation Medicine, Gangnam Severance Hospital, Rehabilitation Institute of Neuromuscular Disease, Yonsei University College of Medicine, Seoul; ${ }^{2}$ Department Rehabilitation Medicine and Research Institute of Rehabilitation Medicine, Yonsei University College of Medicine, Seoul, Korea
\end{abstract}

Objective To evaluate pulmonary functions of patients with amyotrophic lateral sclerosis (ALS), Duchenne muscular dystrophy (DMD), and myotonic muscular dystrophy (MMD) at the onset of ventilatory insufficiency. Methods This retrospective study included ALS, DMD, and MMD patients with regular outpatient clinic followup in the Department of Rehabilitation Medicine at Gangnam Severance Hospital before the application of noninvasive positive pressure ventilation (NIPPV). The patients were enrolled from August 2001 to March 2014. If patients experienced ventilatory insufficiency, they were treated with NIPPV, and their pulmonary functions were subsequently measured.

Results Ninety-four DMD patients, 41 ALS patients, and 21 MMD patients were included in the study. The mean $\mathrm{SpO}_{2}$ was lower in the MMD group than in the other two groups. The mean forced vital capacity (FVC) in the supine position was approximately low to mid $20 \%$ on average in DMD and ALS patients, whereas it was $10 \%$ higher in MMD patients. ALS patients showed a significantly lower FVC in the supine position than in the sitting position. Maximal insufflation capacity, unassisted peak cough flow, maximum inspiratory pressure (MIP), and maximum expiratory pressure (MEP) were significantly higher in MMD group than in the other groups. MEP was significantly the lowest in DMD patients, followed by in ALS, and MMD patients, in order.

Conclusion Disease-specific values of pulmonary function, including FVC, MEP, and MIP, can be accurately used to assess the onset of ventilatory insufficiency in patients with ALS, DMD, and MMD.

Keywords Neuromuscular diseases, Respiratory insufficiency, Duchenne muscular dystrophy, Amyotrophic lateral sclerosis, Myotonic dystrophy

Department of Rehabilitation Medicine and Research Institute of Rehabilitation Medicine, Yonsei University College of Medicine, 50-1 Yonsei-ro, Seodaemun-gu, Seoul 03722, Korea

Tel: +82-2-2227-2994, Fax: +82-2-363-2795, E-mail: iskil98@naver.com

@ This is an open-access article distributed under the terms of the Creative Commons Attribution Non-Commercial License (http://creativecommons.org/ licenses/by-nc/4.0) which permits unrestricted noncommercial use, distribution, and reproduction in any medium, provided the original work is properly cited. Copyright (๑) 2016 by Korean Academy of Rehabilitation Medicine 


\section{INTRODUCTION}

In patients with neuromuscular disease, respiratory muscle weakness and elastic load are responsible for rapid and shallow breathing patterns that lead to chronic $\mathrm{CO}_{2}$ retention [1]. Adequate and timely ventilatory support, such as non-invasive ventilation in secondary hypercapnic ventilatory insufficiency caused by neuromuscular diseases, can reduce respiratory morbidity and mortality. However, it is difficult to detect the point at which $\mathrm{CO}_{2}$ retention begins. The early symptoms of persistent hypercapnia include nightmares, morning headaches, daytime drowsiness, and generalized fatigue, dyspnea at rest, orthopnea, and paradoxical breathing patterns [2-4]. These symptoms are highly subjective and insignificant. Therefore, $\mathrm{CO}_{2}$ retention could be severe by the time the patient complains of such symptoms [5].

Additionally, the ventilation status worsens during nighttime sleeping due to decreased respiratory center function. Even if $\mathrm{CO}_{2}$ retention occurs at night, the arterial blood gas analysis and end-tidal $\mathrm{CO}_{2}\left(\mathrm{EtCO}_{2}\right)$ levels may appear normal during the day in outpatient clinics. Continuous and non-invasive $\mathrm{CO}_{2}$ monitoring during the night is useful for the evaluation of accurate ventilation status and the detection of ventilatory insufficiency; however, this requires hospitalization.

Patients with neuromuscular diseases show restrictive lung disease patterns due to weakness of the respiratory muscles. Previous research has determined values for various pulmonary functions that could be used as screening tools to detect the precise point of ventilatory insufficiency. Specifically, hypercapnia reportedly occurs in patients with neuromuscular diseases when respiratory muscle strength parameters, such as the maximum inspiratory pressure (MIP) and maximum expiratory pressure (MEP), decrease to $<30 \%$ of the normal value, or when the forced vital capacity (FVC) is reduced to below $55 \%$ of the predicted value [6]. Another report suggested that hypercapnia occurs when FVC is $500-700 \mathrm{~mL}$ [2].

However, neuromuscular disease is a broad term that encompasses many syndromes, and these diseases do not show identical conditions at the onset of ventilatory insufficiency. Additionally, there are no broadly accepted disease-specific screening values for ventilatory insufficiency.

Therefore, we included patients with amyotrophic lateral sclerosis (ALS), Duchenne muscular dystrophy (DMD), and myotonic muscular dystrophy (MMD), which are relatively common neuromuscular diseases, to determine clinically relevant pulmonary functions at the onset of ventilatory insufficiency.

\section{MATERIALS AND METHODS}

\section{Subjects}

This retrospective study included ALS, DMD, and MMD patients who were treated with non-invasive positive pressure ventilation (NIPPV) for the first time during hospitalization in the Department of Rehabilitation Medicine at Gangnam Severance Hospital from August 2001 to March 2014. Patients with regular follow-up at the outpatient clinic prior to NIPPV treatment were enrolled and the hospitalization period was determined.

Patients who did not receive a confirmative diagnosis (even if 1 of the 3 diagnoses was clinically suspected) and those who had other accompanying diseases that may have affected respiration, such as Parkinson disease or chronic obstructive pulmonary disease, were excluded. Additionally, patients who were hospitalized immediately at their first visit to the hospital due to previous $\mathrm{CO}_{2}$ retention symptoms or high $\mathrm{EtCO}_{2}$, as well as the patients in whom invasive ventilator was the first interface used for respiration, such as in tracheostomy or intubation, were excluded from the study.

\section{Detection of ventilatory insufficiency and application of NIPPV}

Patients were hospitalized if there were $\mathrm{CO}_{2}$ retention symptoms or if the simplified $\mathrm{EtCO}_{2}$ level measured on the spot was $\geq 40 \mathrm{mmHg}$ than those from regular followups in the outpatient clinic. After hospitalization, capillary $\mathrm{CO}_{2}$ level and oxygen saturation were measured through continuous and non-invasive monitoring of respiration during nighttime sleeping hours. The $\mathrm{CO}_{2}$ partial pressure of the capillary vessels $\left(\mathrm{pCO}_{2}\right)$ was directly and percutaneously measured through the SenTec system (SenTec AG, Therwil, Switzerland) or indirectly measured by the $\mathrm{EtCO}_{2}$ level through the DASH Series (GE Healthcare, Milwaukee, WI, USA). Ventilatory insufficiency was identified as present when the maximum $\mathrm{CO}_{2}$ level was $>40 \mathrm{mmHg}$ or the oxygen saturation was $<95 \%$; NIPPV was then applied [7]. The study was conducted when 
ventilatory insufficiency was present.

\section{Evaluation of pulmonary function}

Pulmonary function was measured immediately after the detection of ventilatory insufficiency. FVC and maximal insufflation capacity (MIC) were measured in the sitting and supine positions using a hand-held spirometer (Micro Medical Ltd., Rochester, Kent, UK). FVC was expressed as a percentage of the predicted normal value $[8,9]$.

In order to measure MIC, the patient was asked to inhale as much air as possible; then, an extra volume of air was delivered by a manual resuscitator bag using either an oronasal mask or a mouthpiece. After holding in the additional air, the patient was asked to exhale as much air as possible into the spirometer.

Peak cough flow (PCF) was measured using a peak-flow meter (Philips Respironics, Guildford, UK). Unassisted PCF (UPCF) was measured by asking the patient to inhale as much air as possible and then cough heavily. Assisted PCF (APCF) was measured with the tester thrusting the patient's abdomen when the patient coughed in the MIC state.

MIP and MEP, which are indicators of the strength of the respiratory muscles, were also measured in the sitting and supine positions using a mouth pressure meter (Micro Medical Ltd.). Measurements were expressed as the percentage of the predicted value, using Wilson and Cooke's data [10].

All measurements were performed at least thrice. The highest positive value of FVC, MIC, UPCF, APCF, and MEP and the lowest negative value for MIP were included in the analysis.

\section{Statistical analysis}

Statistical analysis was performed using SPSS ver. 20.0
(IBM Corp., Armonk, NY, USA). The normality of the distribution of the variables was assessed using the ShapiroWilk test. The Kruskal-Wallis test was used to analyze the differences among the 3 study groups. Using the MannWhitney U test, multiple comparison analyses between 2 different groups were performed with Bonferroni correction as a post-hoc test. Moreover, in each study group, the differences in FVC between the sitting and supine positions were analyzed with a paired t-test. All tests were two-sided, and p-values $<0.05$ were considered statistically significant.

\section{RESULTS}

\section{General characteristics of the study groups}

A total of 156 patients were included in the present study, of which 94 had DMD, 41 had ALS, and 21 had MMD. The mean age of the patient groups was 18.1, 55.9, and 42.7 years, respectively, with significant differences among the 3 groups (Tables 1, 2). There were 17 male patients with ALS (41.5\%) and 11 with MMD (52.4\%) (Table 1).

\section{Continuous monitoring of ventilatory status during nighttime sleep}

The maximal value of $\mathrm{pCO}_{2}$ throughout the continuous monitoring during nighttime sleep showed no significant group-wise differences. The mean saturation of peripheral oxygen was lower in the MMD group than in the other 2 groups. The mean saturation of peripheral oxygen was significantly lower in MMD patients than in DMD patients $(\mathrm{p}=0.045)$.

\section{Evaluation of pulmonary function}

There was a significant difference in FVC in the sitting (FVC_sit(\%)) and supine (FVC_sup(\%)) positions among the 3 groups (Table 2). ALS and MMD patients showed

Table 1. General characteristics of the study groups

\begin{tabular}{lcccc}
\hline & DMD (n=94) & ALS (n=41) & MMD (n=21) & Total (n=156) \\
\hline Age (yr) & $18.1 \pm 3.9$ & $55.9 \pm 10.1$ & $42.7 \pm 10.6$ & $31.4 \pm 18.2$ \\
\hline Sex (male:female) & $94: 0$ & $17: 24$ & $11: 10$ & $122: 34$ \\
\hline FVC_sit (mL) & $951.0 \pm 492.0$ & $1,165.2 \pm 504.3$ & $1,806.5 \pm 825.8$ & $1,124.5 \pm 617.0$ \\
FVC_sup (mL) & $930.2 \pm 498.2$ & $989.5 \pm 451.0$ & $1,703.0 \pm 854.0$ & $1,050.7 \pm 602.2$ \\
\hline
\end{tabular}

Values are presented as mean \pm standard deviation or number.

DMD, Duchenne muscular dystrophy; ALS, amyotrophic lateral sclerosis; MMD, myotonic muscular dystrophy; FVC_ sit, forced vital capacity at sitting position; FVC_sup, forced vital capacity at supine position. 
Table 2. Comparisons of various measurements among the study groups

\begin{tabular}{|c|c|c|c|c|c|c|c|}
\hline & \multirow[b]{2}{*}{ DMD } & \multirow[b]{2}{*}{ ALS } & \multirow[b]{2}{*}{ MMD } & \multicolumn{4}{|c|}{ p-value } \\
\hline & & & & $\begin{array}{l}\text { DMD vs. } \\
\text { ALS }\end{array}$ & $\begin{array}{l}\text { DMD vs. } \\
\text { MMD }\end{array}$ & $\begin{array}{l}\text { ALS vs. } \\
\text { MMD }\end{array}$ & $\begin{array}{l}\text { Among } \\
\text { groups }\end{array}$ \\
\hline Age (yr) & $17.4 \pm 2.5$ & $56.8 \pm 5.3$ & $43.8 \pm 6.6$ & $<0.001$ & $<0.001$ & $<0.001$ & $<0.001$ \\
\hline \multicolumn{8}{|l|}{$\begin{array}{l}\text { Continuous monitoring of ventila- } \\
\text { tor status during sleep time }\end{array}$} \\
\hline Max_CO${ }_{2}(\mathrm{mmHg})$ & $50.3 \pm 3.1$ & $52.7 \pm 5.1$ & $52.0 \pm 6.2$ & 0.666 & 0.177 & 1.000 & 0.127 \\
\hline Mean_SpO ${ }_{2}(\%)$ & $97.0 \pm 1.1$ & $96.0 \pm 1.9$ & $95.0 \pm 3.0$ & 0.357 & 0.045 & 0.453 & 0.022 \\
\hline \multicolumn{8}{|l|}{$\begin{array}{l}\text { Evaluations for pulmonary } \\
\text { function }\end{array}$} \\
\hline FVC_sit(\%) & $23.5 \pm 9.8$ & $31.4 \pm 10.1$ & $39.9 \pm 13.0$ & 0.042 & $<0.001$ & 0.099 & $<0.001$ \\
\hline FVC_sup(\%) & $22.5 \pm 10.4$ & $24.8 \pm 8.4$ & $34.7 \pm 10.0$ & 0.696 & 0.003 & 0.030 & 0.003 \\
\hline p-value (FVC_sit vs. sup) & 0.074 & $<0.001$ & 0.066 & & & & \\
\hline MIC (mL) & $1,540 \pm 329$ & $1,835 \pm 622$ & $2,680 \pm 860$ & 0.141 & $<0.001$ & 0.045 & $<0.001$ \\
\hline UPCF (L/min) & $175 \pm 48$ & $145 \pm 55$ & $260 \pm 65$ & 1.000 & $<0.001$ & 0.003 & $<0.001$ \\
\hline APCFmic (L/min) & $290 \pm 53$ & $270 \pm 87$ & $340 \pm 85$ & 1.000 & 0.093 & 0.456 & 0.120 \\
\hline MIP_sit(\%) & $24.0 \pm 13.4$ & $25.2 \pm 9.0$ & $41.6 \pm 12.2$ & 1.000 & 0.003 & 0.006 & 0.002 \\
\hline MEP_sit(\%) & $15.4 \pm 4.8$ & $26.1 \pm 12.8$ & $37.3 \pm 8.7$ & $<0.001$ & $<0.001$ & 0.252 & $<0.001$ \\
\hline p-value (MIP_sit vs. MEP_sit) & $<0.001$ & 0.666 & 0.051 & & & & \\
\hline MIP_sup(\%) & $25.0 \pm 12.5$ & $23.6 \pm 7.6$ & $35.8 \pm 15.9$ & 1.000 & 0.066 & 0.015 & 0.019 \\
\hline MEP_sup(\%) & $15.4 \pm 5.8$ & $23.7 \pm 11.3$ & $37.0 \pm 8.7$ & 0.012 & $<0.001$ & 0.015 & $<0.001$ \\
\hline p-value (MIP_sup vs. MEP_sup) & $<0.001$ & 0.789 & 0.447 & & & & \\
\hline
\end{tabular}

Values are presented as mean \pm standard deviation.

DMD, Duchenne muscular dystrophy; ALS, amyotrophic lateral sclerosis; MMD, myotonic muscular dystrophy; FVC_ sit(\%), a percent of the predicted value of forced vital capacity at sitting position; FVC_sup(\%), a percent of the predicted value of forced vital capacity at supine position; MIC, maximal insufflation capacity; UPCF, unassisted peak cough flow; APCF, assisted peak cough flow; MIP_sit(\%), a percent of the predicted value of maximum inspiratory pressure at sitting position; MIP_sup(\%), a percent of the predicted value of maximal inspiratory pressure at supine position; MEP_sit(\%), a percent of the predicted value of maximum expiratory pressure at sitting position; MEP_sup(\%), a percent of the predicted value of maximum expiratory pressure at supine position.

significantly higher values of FVC_sit(\%), as compared to the DMD patients. FVC_sit(\%) was $23.5 \% \pm 9.8 \%$ in the DMD group, $31.4 \% \pm 10.1 \%$ in the ALS group, and $39.9 \% \pm 13.0 \%$ in the MMD group ( $\mathrm{p}=0.042$ for DMD vs. ALS; $\mathrm{p}<0.001$ for DMD vs. MMD). This value was also higher in the MMD group than in the ALS group; however, the difference was not statistically significant. The mean FVC_sup(\%) was similar for the DMD and ALS patients, with no significant difference between the 2 groups (FVC_sit(\%) $=22.5 \% \pm 10.4 \%$ for DMD, FVC_sup(\%) $=24.8 \% \pm 8.4 \%$ for ALS). However, the mean FVC_sup $(\%)$ was about $10 \%$ higher in MMD group than in the other 2 groups, which was statistically significant (FVC_sup(\%) $=34.7 \% \pm 10.0 \%$ for MMD). As for FVC by position, ALS patients showed a significantly higher FVC_sit(\%) than
FVC_sup(\%) (FVC_sit(\%) $=31.4 \% \pm 10.1 \%$, FVC_sup(\%)= $24.8 \% \pm 8.4 \%, \mathrm{p}<0.001)$. In DMD and MMD patients, there was no statistically significant difference in FVC between the 2 positions.

MIC and UPCF were higher in MMD patients than in the other patient groups, whereas APCF showed no significant difference among the 3 groups.

MIP and MEP were also significantly higher in MMD patients than in the other groups. MEP was significantly lowest in DMD patients, followed by ALS patients, and MMD patients, in order.

In DMD patients, MEP was significantly lower than MIP regardless of the position (MEP in sitting position $=15.4 \pm 4.8 \mathrm{mmHg}$, MIP in sitting position $=24.0 \pm 13.4$ $\mathrm{mmHg}, \mathrm{p}<0.001$; MEP in supine position $=15.4 \pm 5.8$ 
$\mathrm{mmHg}$, MIP in supine position $=25.0 \pm 12.5 \mathrm{mmHg}$, $\mathrm{p}<0.001)$. In patients with MMD, MEP was lower than MIP in the sitting position but not in the supine position; however, the difference was not statistically significant. Additionally, MEP and MIP showed no significant differences in ALS patients.

\section{DISCUSSION}

Neuromuscular disease refers to a variety of diseases that adversely affect the nerves and muscles and may indicate multiple diagnoses, with a range of potential clinical courses according to each diagnosis. The mechanism of ventilatory insufficiency varies according to the specific type of neuromuscular disease. By comparing the pulmonary functions at the onset of ventilatory insufficiency in different types of neuromuscular diseases, we determined the distinctive characteristics for each of these diseases and the specific points to consider during ventilator application.

The results of this study suggested that patients should be closely observed when their pulmonary function values fall below the specific cutoff values. In case of DMD, ventilatory insufficiency occurred on average when FVC and MIP were approximately $20 \%-25 \%$ and MEP was about $15 \%$ of the patient's normal values. Therefore, close observation is required if FVC, MEP, and MIP drop below $40 \%, 30 \%$, and $40 \%$ of the patient's normal values, respectively, in either the supine or sitting positions. For ALS, ventilatory insufficiency occurred when the supine FVC, MEP, and MIP were approximately $20 \%-25 \%$ of the patient's normal values; thus, close observation is needed if FVC, MEP, and MIP drop below $40 \%$ of the patient's normal values in the supine position. Ventilatory insufficiency began in MMD patients when the supine FVC was about $35 \%$ of the normal values, hence these patients require closer attention than DMD or ALS patients. Thus, close observation is needed for MMD patients when the supine FVC drops below $50 \%$ of the patient's normal values.

Unlike the results of our study, a previous study reported that hypercapnia occurs when respiratory muscle strength is $<30 \%$ and FVC drops below $55 \%$ of the predicted value in patients with neuromuscular diseases [6]. However, the previous study included patients with nonspecific neuromuscular diseases; thus, the difference in the patients' homogeneity may have led to the conflicting results.

In the present study, MMD patients showed earlier ventilatory insufficiency than the other 2 patient groups despite their well-preserved pulmonary functions. This result is in agreement with that of a previous study, which suggested that ventilatory insufficiency could occur when respiratory muscle weakness is not pronounced in MMD patients [11]. Our study also showed that many parameters other than FVC (e.g., MEP, MIP, or PCF) are better preserved in MMD patients, as compared with the other 2 patient groups.

There are 2 main reasons other than respiratory muscle weakness for such disproportionate rates of ventilatory insufficiency in MMD patients. First, delayed relaxation may occur in the respiratory muscles as it does in other muscles in MMD patients. One study verified the delayed relaxation of the diaphragm using fluoroscopy [12], and another confirmed the myotonic movement of the respiratory muscles, including the diaphragm, through electromyography $[11,13]$. Another possibility is that MMD patients have trouble with central respiratory control [14]. One study suggested that the high incidence of ventilatory insufficiency in MMD patients is related to a decreased hypoxic ventilatory response due to an underlying neurogenic deficit [15]. Another study suggested that neuronal loss in the medullary arcuate nucleus is associated with hypoventilation in myotonic dystrophy [16].

However, there has been insufficient research on whether the myotonic movement of respiratory muscles actually causes ventilatory insufficiency; and there are still many controversies concerning the main factors involved. In the present study, the mean saturation of peripheral oxygen tended to be lower in MMD patients than the other groups, which indicates the possibility of sleep apnea. Further evaluation including polysomnography is needed.

Although sitting FVC was higher in ALS patients, there were no significant differences in the predictive value of supine FVC between ALS and DMD patients. This indicates that ALS patients exhibit significant differences in FVC between the sitting and supine positions, unlike those of the other patient groups. FVC also tends to be lower in the supine position for healthy people because the abdominal contents press on the diaphragm in this position, thus increasing the amount of pulmonary circu- 
lation and reducing the volume of air entering the thorax [17]. However, this lower value of supine FVC is greater in ALS patients because of the weakness of the diaphragm $[18,19]$. Therefore, FVC should be measured in the supine position in ALS patients. Loss in diaphragmatic strength is spared until the very late stages of DMD $[20,21]$, and consequently, the fall in supine FVC is not generally obvious in DMD patients.

Lastly, MEP was less than MIP in both DMD and MMD patients in the sitting position. Previous studies have revealed that the first indication of pulmonary dysfunction in DMD and MMD populations is a decline in MEP [14,22,23]. Thus, MEP should be considered when screening for ventilatory insufficiency in DMD and MMD patients.

The limitations of this study were that the patient group included only 3 diseases and a relatively small number of patients were enrolled in the MMD group, as compared with the other 2 groups. Further studies are needed to reveal the pulmonary functions and mechanisms contributing to ventilatory insufficiency in these and other types of neuromuscular diseases.

In conclusion, pulmonary functions at the onset of ventilatory insufficiency differ in ALS, DMD, and MMD patients according to the different characteristics of each disease. Disease-specific pulmonary values (including FVC, MEP, and MIP) could be used to accurately screen for ventilatory insufficiency.

\section{CONFLICT OF INTEREST}

No potential conflict of interest relevant to this article was reported.

\section{REFERENCES}

1. Misuri G, Lanini B, Gigliotti F, Iandelli I, Pizzi A, Bertolini MG, et al. Mechanism of $\mathrm{CO}(2)$ retention in patients with neuromuscular disease. Chest 2000;117:447-53.

2. Baydur A, Gilgoff I, Prentice W, Carlson M, Fischer DA. Decline in respiratory function and experience with long-term assisted ventilation in advanced Duchenne's muscular dystrophy. Chest 1990;97:8849.

3. Finder JD, Birnkrant D, Carl J, Farber HJ, Gozal D, Ian- naccone ST, et al. Respiratory care of the patient with Duchenne muscular dystrophy: ATS consensus statement. Am J Respir Crit Care Med 2004;170:456-65.

4. Simonds AK. Recent advances in respiratory care for neuromuscular disease. Chest 2006;130:1879-86.

5. Rochester DF, Esau SA. Assessment of ventilatory function in patients with neuromuscular disease. Clin Chest Med 1994;15:751-63.

6. Braun NM, Arora NS, Rochester DF. Respiratory muscle and pulmonary function in polymyositis and other proximal myopathies. Thorax 1983;38:616-23.

7. Bach JR, Alba AS. Noninvasive options for ventilatory support of the traumatic high level quadriplegic patient. Chest 1990;98:613-9.

8. Morris JF. Spirometry in the evaluation of pulmonary function. West J Med 1976;125:110-8.

9. da Costa JL. Pulmonary function studies in healthy Chinese adults in Singapore. Am Rev Respir Dis 1971;104:128-31.

10. Wilson SH, Cooke NT, Edwards RH, Spiro SG. Predicted normal values for maximal respiratory pressures in caucasian adults and children. Thorax 1984;39:535-8.

11. Rimmer KP, Golar SD, Lee MA, Whitelaw WA. Myotonia of the respiratory muscles in myotonic dystrophy. Am Rev Respir Dis 1993;148(4 Pt 1):1018-22.

12. Benaim S, Worster-Drought C. Dystrophia myotonica with myotonia of the diaphragm causing pulmonary hypoventilation with anoxaemia and secondary polycythaemia. Med Illus 1954;8:221-6.

13. Jammes Y, Pouget J, Grimaud C, Serratrice G. Pulmonary function and electromyographic study of respiratory muscles in myotonic dystrophy. Muscle Nerve 1985;8:586-94.

14. Serisier DE, Mastaglia FL, Gibson GJ. Respiratory muscle function and ventilatory control. I: in patients with motor neurone disease. II: in patients with myotonic dystrophy. Q J Med 1982;51:205-26.

15. Carroll JE, Zwillich CW, Weil JV. Ventilatory response in myotonic dystrophy. Neurology 1977;27:1125-8.

16. Ono S, Takahashi K, Kanda F, Jinnai K, Fukuoka Y, Mitake S, et al. Decrease of neurons in the medullary arcuate nucleus in myotonic dystrophy. Acta Neuropathol 2001;102:89-93.

17. Bourke SC, Bullock RE, Williams TL, Shaw PJ, Gibson GJ. Noninvasive ventilation in ALS: indications and effect on quality of life. Neurology 2003;61:171-7. 
18. Varrato J, Siderowf A, Damiano P, Gregory S, Feinberg D, McCluskey L. Postural change of forced vital capacity predicts some respiratory symptoms in ALS. Neurology 2001;57:357-9.

19. Lechtzin N, Wiener CM, Shade DM, Clawson L, Diette GB. Spirometry in the supine position improves the detection of diaphragmatic weakness in patients with amyotrophic lateral sclerosis. Chest 2002;121:436-42.

20. Khirani S, Ramirez A, Aubertin G, Boule M, Chemouny C, Forin V, et al. Respiratory muscle decline in Duchenne muscular dystrophy. Pediatr Pulmonol 2014;49:473-81

21. Stedman HH, Sweeney HL, Shrager JB, Maguire
HC, Panettieri RA, Petrof B, et al. The mdx mouse diaphragm reproduces the degenerative changes of Duchenne muscular dystrophy. Nature 1991;352:5369.

22.Hahn A, Bach JR, Delaubier A, Renardel-Irani A, Guillou C, Rideau Y. Clinical implications of maximal respiratory pressure determinations for individuals with Duchenne muscular dystrophy. Arch Phys Med Rehabil 1997;78:1-6.

23. Begin P, Mathieu J, Almirall J, Grassino A. Relationship between chronic hypercapnia and inspiratorymuscle weakness in myotonic dystrophy. Am J Respir Crit Care Med 1997;156:133-9. 